\title{
Diabetic Ketoacidosis Misdiagnosed and Managed as Typhoid lleal Perforation in a Ghanaian Child: A Case Report
}

\author{
Emmanuel Ameyaw, F.W.A.C.P. ${ }^{1}$, Alhassan Abdul-Mumin, F.G.C.P.S. ${ }^{2}$, \\ Abiboye Cheduko Yifieyeh, F.W.A.C.S. ${ }^{3}$, Akua Afriyie Ocran, M.W.A.C.P. ${ }^{1}$, \\ Naana Ayiwa Wereko Brobbey, M.W.A.C.P. ${ }^{1}$
}

'Department of Child Health, School of Medicine and Dentistry, Kwame Nkrumah University of Science and Technology, Kumasi, Ashanti Ayigya, Ghana.

${ }^{2}$ Department of Paediatrics and Child Health, School of Medicine and Health Sciences, University for Development Studies, Tamale, Northern Kukuo, Ghana.

${ }^{3}$ Department of Surgery, School of Medicine and Dentistry, Kwame Nkrumah University of Science and Technology, Kumasi, Ashanti Ayigya, Ghana.

Received 25 January 2021 • Revised 27 April 2021 • Accepted 17 May $2021 \bullet$ Published online 5 July 2021

\begin{abstract}
:
We report on a 15-year-old Ghanaian boy, who presented to a district hospital with diabetic ketoacidosis (DKA) but was diagnosed wrongly as a small bowel perforation due to typhoid fever. He presented with weakness, poor feeding, vomiting, and severe abdominal pain. General examination revealed a lethargic, drowsy, and severely dehydrated patient. His abdomen was diffusely tender more in the right ileac fossa. Investigations including complete blood count, blood smear for malaria parasites, urinalysis, and abdominal ultrasound yielded unremarkable results. Hemoglobin level was 12.4 grams per decilitre. He was diagnosed as typhoid ileal perforation and laparotomy was done, but the bowels and all other intra-abdominal organs were found to be normal. A blood glucose test was done intraoperatively, and it found very high hyperglycaemia. This led to the suspicion of DKA, which was confirmed through urine dipstick testing for high urine ketones and a high glycated hemoglobin (HbA1C) test result. He was then treated for DKA, which resolved on the second day of admission, but the surgical wound healed after twelve days.
\end{abstract}

Contact: Emmanuel Ameyaw, F.W.A.C.P.

Department of Child Health, School of Medicine and Dentistry,

Kwame Nkrumah University of Science and Technology, Kumasi, Ashanti Ayigya, Ghana.

E-mail: ekameyaw@yahoo.com

This is an open access article under the CC BY-NC-ND license

(http://www.jhsmr.org/index.php/jhsmr/about/editorialPolicies\#openAccessPolicy).

J Health Sci Med Res 2022;40(2):223-227 doi: 10.31584 /jhsmr.2021825 www.jhsmr.org 
Our account emphasizes the need for testing for blood glucose in critically ill children and adolescents with suspected severe infections or acute medical conditions that require admission or surgical intervention. This owes to the fact that the clinical features of undiagnosed diabetes, especially if it progresses to DKA, are similar to those of infections and disorders managed via surgical treatment, which are rather more common in Ghana and Africa.

Keywords: diabetic ketoacidosis, laparotomy, misdiagnosis, typhoid perforation

\section{Introduction}

Diabetic ketoacidosis (DKA) is a medical emergency and requires an immediate and prompt diagnosis and early management. ${ }^{1,2}$ However, the misdiagnosis of DKA is common among physicians in Africa; ${ }^{1,3}$ yet it occurs in advanced countries with well-resourced health systems. ${ }^{4,5}$ In Africa, poor healthcare infrastructure, poverty, ignorance, heavy infectious disease burdens, limited training in type1 diabetes (T1D) for health workers, lack of community awareness, and insufficient support from local governments for children living with diabetes mellitus compound the misdiagnosis of DKA. ${ }^{1,3,6,7}$

T1D is a heterogeneous disorder characterized by the destruction of beta cells of pancreas, resulting in total insulin dificiency. ${ }^{8}$ Affected patients, therefore, need daily insulin treatment to survive. ${ }^{9}$ DKA occurs due to a relative or complete lack of insulin, resulting in the intracellular starvation of insulin-dependent tissues such as muscle, liver, and adipose tissues. This leads to the release of counter-regulatory hormones like glucagon, catecholamines, cortisol, and growth hormone, which induce fat and protein breakdown, glucose production in kidneys and liver, and the hepatic oxidation of fatty acid to produce ketone bodies. ${ }^{3,10}$ The accumulation of ketone bodies in the blood produce the clinical features of DKA such as weakness, nausea, vomiting, abdominal pain, fast respiration, lethargy, loss of consciousness, and coma. ${ }^{2}$ These clinical features are also found in acute infections such as cerebral malaria, meningitis, pneumonia, gastroenteritis, and typhoid fever. ${ }^{11-13}$
Therefore, the misdiagnosis of DKA as an infectious disease is possible and probably more common in Africa than has been reported. Nevertheless, the failure to recognize the clinical features of T1D mellitus and DKA occurs even in well-resourced countries. ${ }^{4,5,7,14}$ Rwiza et al. ${ }^{14}$ reported a misdiagnosis of DKA among children in most hospitals in Tanzania. The wrongly diagnosed conditions included malaria, urinary tract infection, diarrhoea, and pneumonia. In Ghana, Kratzer ${ }^{7}$ found out that all categories of health workers made wrong diagnoses such as malaria and typhoid fever instead of diabetes because of the similarities in clinical features between diabetes and these infections. A wrong diagnosis in such cases has serious implications because the misdiagnosed patient would be managed inappropriately, which could lead to complications and even mortality.

We report a case of a 15-year-old boy with DKA who presented with poor feeding, vomiting and severe abdominal pain to a district hospital in Ghana and was wrongly diagnosed as a case of perforated ileum due to typhoid fever, for which laparotomy was performed. To our knowledge, no such a case has been reported previously in literature.

\section{Case Report}

A 15-year-old boy was sent to a district hospital in 2018 because of weakness, poor feeding and vomiting for 6 days, and severe abdominal pain of two days. He did not have any previous medical history or family history of diabetes mellitus. However, he had been admitted two times 
within three months prior to presentation and was treated for malaria and typhoid fever. He spent an average of 5 days between admission and discharge from the ward during those episodes. On examination, he appeared wasted (his weight was 37 kilograms, i.e., below the $3^{\text {rd }}$ percentile, and his height was $162 \mathrm{~cm}$, or above the $10^{\text {th }}$ percentile), lethargic, drowsy, breathless, and dehydrated; the level of dehydration was estimated to be $10.0 \%$. The abdomen was diffusely tender and more in the right ileac fossa. His blood pressure was 90/60 millimeter of mercury, and his pulse was 100 beats per minute. The abdominal ultrasound was inconclusive, the blood smear for malaria parasites was negative, and the levels of liver enzymes, urea, and creatinine were normal. The complete blood count revealed leucocytosis. Investigations for electrolytes and blood gases could not be done. He was diagnosed with typhoid ileal perforation and arranged for laparotomy. He was initially resuscitated with one litre each of normal saline with 5.0\% dextrose and Ringer's lactate solutions over two hours and put on intravenous (IV) metronidazole 500 milligram (mg) three times daily and gentamycin $80 \mathrm{mg}$ two times daily. During laparotomy, no intestinal perforations were identified. The blood glucose level was then checked, and it was $>33.3$ millimol per liter $(\mathrm{mmol} / \mathrm{L})$. Moreover, the urine ketone level was found to be 4+ via urine dipstick testing, and the glycated hemoglobin was $13.8 \%$. The abdomen was then sutured in layers, and finally, the patient was sent to the recovery ward as a case of DKA. Further past medical history inquire revealed that he had had polyuria, polydipsia, polyphagia, and enuresis two months prior to presentation.

After recovery from anaesthesia, he was sent to the ward and managed as a case of DKA according to the International Society for Pediatric and Adolescent Diabetes's protocol for the management of DKA. ${ }^{15}$ He was given an extra five litres of IV fluids (normal saline and normal saline with $10.0 \%$ dextrose) over 44 hours at maintenance rate. Furthermore, he was given short-acting human insulin at a dose of 0.1 units per kilogram (3.7 units per hour). Potassium chloride was also started at the same time as the short-acting human insulin at a dose of $40 \mathrm{mmol} /$, administered via the IV fluids. He was monitored every hours for blood glucose, every two hours for urine ketones via a urine dipstick test, and every 4 hours for vital signs. His blood glucose level dropped to $14 \mathrm{mmol} / \mathrm{L} 8$ hours after starting the short-acting human insulin. The IV normal saline solution was then changed to IV normal saline with $10.0 \%$ dextrose and continued along with short-acting human insulin until all ketones cleared from the urine. DKA resolved on the second day of admission, but the surgical wound healed after twelve days. He was discharged on day thirteen on a multiple-dose insulin injection regimen

Table 1 History and investigations of diabetic ketoacidosis in children and adolescents

\begin{tabular}{lll}
\hline Clinical presentation & Physical examination & Investigation \\
\hline Polyuria & Wasted & High blood glucose $\geq 11.1 \mathrm{mmol} /$ \\
Polyphagia & Dehydration & Positive urine ketones \\
Polydipsia & Kussmaul breathing & Positive blood ketones \\
Wasting & Acetone breath & Venous $\mathrm{pH}<7.25$ or $\mathrm{Arterial} \mathrm{pH}<7.3$ \\
Weakness & Shock & Bicarbonate $<15 \mathrm{mmol} / \mathrm{L}$ \\
Lethargy & Coma & \\
Nausea & & \\
Vomiting & & \\
Abdominal pains & \\
Difficulty in breathing & \\
\hline
\end{tabular}


using both short- and intermediate-acting insulin. During admission, his blood glucose was maintained within the target range. ${ }^{16}$

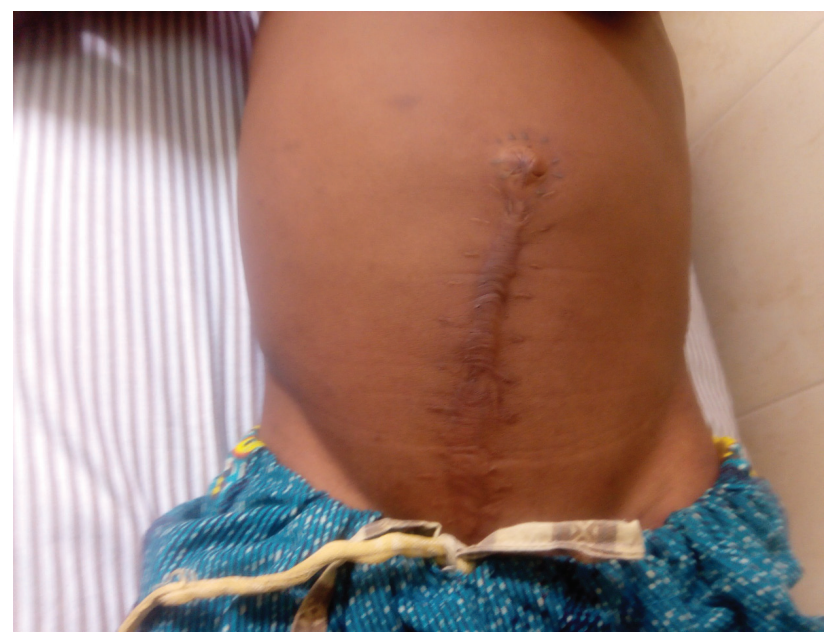

Figure 1 A 15-year-old boy with a surgical scar from laparotomy

\section{Discussion}

Failure to diagnose DKA in children and adolescents can have lethal consequences such as neurological complications and death. ${ }^{3,14}$ Misdiagnoses of T1D and DKA occur more in resource-limited countries, although they also occur in rich countries with well-organized health systems. ${ }^{1,2,4}$ DKA is a medical emergency that demands prompt diagnosis and immediate management. Any delay in treatment can have detrimental consequences on the patient. Our patient was misdiagnosed as a case of typhoid ileal perforation because he had the clinical features of bodily pains, vomiting, and abdominal pain that was more pronounced in the right iliac fossa region ${ }^{13}$, which are also clinical features that characterise typhoid fever. Moreover, especially at the district hospital level, typhoid infection among children and adolescents is more commonly seen in Ghana than diabetes. ${ }^{13,18}$
The misdiagnosis of DKA could be dangerous because the misdiagnosed patient would undergo wrong management and, hence, may develop further complications and even die. Such a medical error is facilitated by the similarities in clinical features between DKA and typhoid perforation. ${ }^{1,2,13}$ Besides, many district hospitals in Ghana have poor laboratory support to enable the correct diagnosis of such conditions. Typically, district hospitals can only do basic investigations such as the rapid diagnostic test for malaria, the blood smear test for malaria, and the urine dipstick test, but they lack the facilities for performing cultures and sensitive electrolytes and biochemistry tests such as those that investigate liver enzymes, the blood urea nitrogen level, and blood gases. Unfortunately, the patient was wrongly operated upon; the proper management for his condition does not require an operation. This misdiagnosis could have possibly been avoided if the blood glucose test had been done before considering the surgical intervention. Furthermore, the patient was dehydrated, and the initial fluid resuscitation included dextrose administration. It is very likely that this worsened the clinical presentation.

A more effective education of both health professionals and general communities, including schools, about the clinical features of diabetes and DKA among children would be a significant step in the right direction in improving awareness ${ }^{1,2,4,5}$ and ensuring that children and adolescents suspected of having diabetes are sent to hospitals for immediate assessment, which would enable the early diagnosis and treatment of these medical conditions. It is also important that local governments support the health system and strengthen the health infrastructure by providing adequate laboratory support, glucometers and strips for screening and monitoring insulin level, and insulin supplies for the early treatment of confirmed cases. Moreover, systems to monitor and follow up such patients in order to ensure adherence to glycaemic targets and prevent complications should also be in place. 


\section{Conclusion}

DKA among children and adolescents can easily be misdiagnosed as an infection because of the similarities in clinical presentations between such conditions. It is, therefore, important that all doctors, irrespective of discipline and level of practice, perform blood glucose tests on all children and adolescents who present to health facilities with acute medical conditions.

\section{Acknowledgement}

The authors are grateful to the patient and his family for allowing them to publish the data related to this case report.

\section{Funding sources}

This research did not receive any financial support.

\section{Conflict of interest}

All authors declare no conflicts of interest.

\section{References}

1. Atkilt HS, Turago MG, Tegegne BS. Clinical Characteristics of Diabetic Ketoacidosis in Children with Newly Diagnosed Type 1 Diabetes in Addis Ababa, Ethiopia: A Cross-Sectional Study. Plos One 2017;12:e0169666

2. Perilli G, Saraceni C, Daniels MN, Ahmad A. Diabetic Ketoacidosis: A Review and Update. Curr Emerg Hosp Med Rep 2013;1:10-7

3. Murunga AN, Owira PM. Diabetic ketoacidosis: an overlooked child killer in sub-Saharan Africa? Trop Med Int Health 2013; 18:1293-422.

4. Sundaram PCB, Day E, Kir JMW. Delayed diagnosis in type diabetes mellitus. Arch Child 2009;94:151-2.

5. Kear C, Fisher T, Westwood E, Sachdev P, Denvi L. Delayed Diagnosis of Type 1 Diabetes Mellitus in Children. Arch Child 2016;101(Suppl 1):A103-4.

6. Rabkin M, Melaku Z, Bruce K, Reja A, Koler A, Tadesse Y, Kamiru HN, Sibanyoni LT and El-Sadr W. Strengthening Health
Systems for Chronic Care: Leveraging HIV Programs to Support Diabetes Services in Ethiopia and Swaziland. J Trop Med 2012. doi:10.1155/2012/137460.

7. Kratzer J. Structural barriers to coping with Type 1 Diabetes Mellitus in Ghana: Experiences of diabetic youth and their families. Ghana Med J 2012;46:39-45.

8. Shields BM, Peters JL, Cooper C, Powell RJ, Knight BA, Hyde C, Hattersley AT. Identifying clinical criteria to predict Type 1 diabetes, as defined by absolute insulin deficiency: a systematic review protocol. BMJ Open 2012;2:1-5.

9. Silverstein J, Klingensmith G, Copeland K, Plotnick, Kaufman F, Laffel L, Deeb L, Grey M et al. Care of Children and Adolescents with Type 1 Diabetes. Diabetes Care 2005;28: 186-212.

10. Wolfsdorf J, Glaser N, Sperling MA. Diabetic Ketoacidosis in Infants, Children, and Adolescents. Diabetes Care 2006;29: 1150-9.

11. Misra UK, Kalita J, Prabhakar S, Chakravarty A, Kochar D, Nair PP. Cerebral malaria and bacterial meningitis. Ann Indian Acad Neurol 2011;14:S35-9.

12. Harris M, Clark J, Cooke N, Fletcher P, Harnden A, Mckean M, et al. British Thoracic Society Guidelines for the Management of Community Acquired Pneumonia in Childhood. Thorax 2011; 66:ii1-23.

13. Kuubiere CB, Mogre V, Majeed SF and Alhassan A. Typhoid intestinal perforations in Northern Ghana. Al Ameen $\mathrm{J}$ M Edical C lence 2014;7:251-6.

14. Rwiza HT, Swai ABM, McLarty DG. Failure to diagnose diabetic ketoacidosis in Tanzania. Diabet Sci 1986;3:181-3.

15. Wolfsdorf J, Glaser N, Agus M, Fritsch M, Hanas R, Rewers A, Sperling MA, Codner E. ISPAD Clinical Practice Consensus Guidelines 2018: Diabetic ketoacidosis and the hyperglycaemic hyperosmolar state. Pediatric Diabetes 2018; 19 (Suppl 27):155-77

16. Ogle G, Middlehurst A, Silink M, Hanas R. Pocketbook for Management of Diabetes in Childhood and Adolescence in Under-resourced Country. $2^{\text {nd }}$ ed. Brussels: International Diabetes Federation; 2017.

17. Espinoza LMC, Nichols C, Adu-Sarkodie Y. Variations of Invasive Salmonella Infections by Population Size in Asante Akim North Municipal, Ghana. Clin Infect Dis 2016;62:S17-22. 